\title{
ECG Interpretation: Clinical Relevance, Challenges, and Advances
}

\author{
Nikita Rafie ${ }^{1}$, Anthony H. Kashou ${ }^{2, *}$ and Peter A. Noseworthy ${ }^{2}$ \\ 1 Department of Medicine, Mayo Clinic, 200 First Street SW, Rochester, MN 55905, USA; rafie.nikita@mayo.edu \\ 2 Division of Cardiovascular Diseases, Mayo Clinic, 200 First Street SW, Rochester, MN 55905, USA; \\ noseworthy.peter@mayo.edu \\ * Correspondence: kashou.anthony@mayo.edu
}

Citation: Rafie, N.; Kashou, A.H.; Noseworthy, P.A. ECG Interpretation: Clinical Relevance, Challenges, and Advances. Hearts 2021, 2, 505-513. https://doi.org/10.3390/ hearts2040039

Academic Editor: Peter Macfarlane

Received: 21 September 2021

Accepted: 20 October 2021

Published: 2 November 2021

Publisher's Note: MDPI stays neutral with regard to jurisdictional claims in published maps and institutional affiliations.

Copyright: (c) 2021 by the authors. Licensee MDPI, Basel, Switzerland. This article is an open access article distributed under the terms and conditions of the Creative Commons Attribution (CC BY) license (https:// creativecommons.org/licenses/by/ $4.0 /)$.

\begin{abstract}
Since its inception, the electrocardiogram (ECG) has been an essential tool in medicine. The ECG is more than a mere tracing of cardiac electrical activity; it can detect and diagnose various pathologies including arrhythmias, pericardial and myocardial disease, electrolyte disturbances, and pulmonary disease. The ECG is a simple, non-invasive, rapid, and cost-effective diagnostic tool in medicine; however, its clinical utility relies on the accuracy of its interpretation. Computer ECG analysis has become so widespread and relied upon that ECG literacy among clinicians is waning. With recent technological advances, the application of artificial intelligence-augmented ECG (AI-ECG) algorithms has demonstrated the potential to risk stratify, diagnose, and even interpret ECGs-all of which can have a tremendous impact on patient care and clinical workflow. In this review, we examine (i) the utility and importance of the ECG in clinical practice, (ii) the accuracy and limitations of current ECG interpretation methods, (iii) existing challenges in ECG education, and (iv) the potential use of AI-ECG algorithms for comprehensive ECG interpretation.
\end{abstract}

Keywords: electrocardiogram; ECG interpretation; artificial intelligence; machine learning

\section{Introduction}

Since its development over a century ago, the ECG remains the cornerstone of cardiovascular screening, evaluation, and diagnosis. The ECG is one of the most widely used tools in medicine today-nearly 200 million ECGs are recorded annually worldwide [1]. While the ECG tracing itself has remained relatively unchanged since its inception, our ability to leverage the "humble" ECG to detect and diagnose various pathologies continues to evolve [2]. Its use is imperative in the evaluation and management of an array of cardiovascular diseases, including arrhythmias, pericardial and myocardial disease, as well as many non-cardiac conditions including electrolyte disturbances and pulmonary disease among many others. Advances in cardiology have been accelerated by technology, and the ECG is at the center of this dependency [3].

The ECG has enabled countless advances in the field of cardiology, bearing witness to the genesis of entire subspecialties. For instant, prior to the development of the ECG, arrhythmias were poorly understood but the ECG is now central to the burgeoning field of cardiac electrophysiology [2]. Similarly, the ECG facilitated monumental strides in the recognition, management, and treatment of acute myocardial infarction (MI) [2]. Our reliance on the ECG to identify life-threatening arrhythmias and acute MIs has become so widespread and critically important that it has become part of the core curriculum for most medical trainees.

Over the last few decades, computing power and digitized data availability have made exponential gains. This has led to the application of artificial intelligence (AI) to the ECG. In recent years, the use of AI-enabled ECG (AI-ECG) algorithms for various risk stratification, diagnostic evaluation, and clinical interpretation have emerged. Researchers have shown some algorithms to be capable of rhythm identification [4] and even perform 
comprehensive 12-lead ECG interpretation [5]. In fact, the accuracy appears to be better than that of the currently implemented ECG software [5].

In this review, we examine (i) the utility and importance of the ECG in clinical practice, (ii) the accuracy and limitations of current ECG interpretation methods, (iii) existing challenges in ECG education, and (iv) the potential use of AI-ECG algorithms for comprehensive ECG interpretation.

\section{ECG and Its Clinical Utility}

The expansive application of the ECG has been well established since its invention and continues to evolve over a century later. Its clinical utility includes, but is not limited to, the detection of acute and chronic myocardial injury, cardiac arrhythmias, structural heart disease, and inflammatory processes (e.g., pericarditis) [3,6-8]. In addition, its use spans throughout nearly all specialties (e.g., cardiology, emergency medicine, internal medicine) and clinical settings (e.g., emergency department, primary care clinic, intensive care unit) in medicine. The ECG is also used to assess whether a medication is safe to prescribe, monitor the effects of a drug on the heart, predict the risk of life-threatening arrhythmias, evaluate for coronary artery disease, and even determine if implantation of a device would improve cardiac function and quality of life.

One of the first clinical applications of the ECG that directly impacted patient care was its ability to identify acute myocardial injury [2]. This helped clinicians to also differentiate cardiac chest pain from non-cardiac chest pain mimickers. Studies comparing various ECG patterns in humans and animals following myocardial infarction led to recognition of ST-segment elevation patterns in patients with myocardial infarction [2]. The reproducible myocardial injury patterns have enabled computer program algorithms to identify STsegment elevation myocardial infarctions (STEMIs). Furthermore, the concept of waveform changes associated with MI and myocardial injury have advanced the utility of the ECG to detect demand ischemia (e.g., exercise-induced ischemia before an episode of acute myocardial infarction) [3]. With this advancement, coronary artery disease and its impact can be detected before a potentially fatal event, leading to improvement in cardiac morbidity and overall mortality.

Arrhythmia detection is one of the most common clinical uses of the ECG. The identification of atrial fibrillation on the ECG remains a key diagnostic component that alters patient management [2]. The ECG also aids in differentiating supraventricular and ventricular arrhythmias [9]. In addition to rhythm detection, the ECG can localize the origin of a rhythm in many instances [3]. This information can aid in patient care (e.g., accessory pathway ablation of an accessory pathway in Wolff-Parkinson-White syndrome).

The ECG can detect structural changes within the heart (e.g., atrial enlargement and ventricular hypertrophy). The identification of left atrial enlargement may help identify patients at risk for developing atrial fibrillation, whereas the presence of left ventricular hypertrophy may identify patients at greater risk of cardiovascular disease from systemic hypertension. In fact, the changes in electrical conduction in a hypertrophied myocardium can help prognosticate cardiovascular disease in patients with hypertension [6]. The diagnostic ECG criteria for ventricular hypertrophy are rather straightforward, which has made it a programmable feature for computer interpretation algorithms to identify. While many ECG criteria for structural abnormalities are rather nonspecific, such findings may direct a clinician towards more aggressive treatment of chronic comorbidities (e.g., hypertension treatment if left ventricular hypertrophy is evident) or further diagnostic evaluation (e.g., echocardiogram).

The ECG can also aid in the diagnosis, recurrence, and resolution of inflammatory conditions, namely, pericarditis. Early and accurate diagnosis and initiation of treatment of pericarditis is imperative in decreasing recurrence and demand of resources from complications [8]. Following the diagnosis and initiation of treatment, the dynamic ECG changes can aid in monitoring for resolution [8]. 
Despite advances in imaging modalities, the ECG remains one of the most rapid and non-invasive tools to aid in the diagnosis of pericardial effusions and cardiac tamponade. The sensitivity of ECG in detecting cardiac tamponade remains quite low despite advances in interpretation so it cannot be used to rule out cardiac tamponade clinically. However, the high specificity of these ECG changes makes the ECG, in conjunction with clinical correlation, a key tool in ruling in cardiac tamponade [7]. As with all other cardiovascular diseases, the ECG can be used to augment clinical suspicion without requiring advanced imaging or invasive procedures, again emphasizing the importance of diagnosis for rapid and accurate diagnosis and treatment.

The simple, non-invasive, rapid, and cost-effective nature of obtaining an ECG allows for its widespread use and powerful diagnostic abilities in medicine. However, the story behind its interpretation is not the same as its simple and routine acquisition.

\section{The Advent and Consequences of Computer-Aided ECG Interpretation}

Major technological, imaging, and procedural advances have flourished across multiple disciplines in medicine over the last century, and the interpretation of the ECG is no exception. Over 50 years ago, computer analysis of the ECG was introduced to extract, analyze, and interpret ECGs [4]. The overarching goals were to improve interpretation accuracy, efficiency, and clinical workflow. What once required knowledge and detailed analysis was now being replaced by computer software programs. The computer processing and analyzing of ECGs has become a mainstay in many clinical settings even to this day. However, this is certainly not without consequences.

Over the years, medical providers have become more reliant on the computerized ECG interpretation for aiding in clinical decision making. Unfortunately, this is an issue given the notorious inaccuracies associated with such ECG interpretation software. Perhaps an inadvertent byproduct of such reliance is less focus around ECG education across medical training programs. This has merely reduced ECG competency to a small minority of skilled providers such that the skill of ECG interpretation has become a lost art.

Countless studies have demonstrated significant inaccuracies and limitations of computer ECG interpretation software [10]. This is a major problem given the reliance of medical providers on the software and the subsequent direct impact it can have on patient care. Despite attempts to improve the accuracy of computerized ECG interpretation algorithms, the final ECG interpretation continues to rely on a physician's over-read [10-13]. In addition, even with efforts to standardize ECG interpretation in the United States and Europe, there has yet to be an all-encompassing internationally accepted standard for computerized ECG interpretation [14].

\section{ECG Interpretation Accuracy and Limitations}

Although the ECG is central to practice, some have argued that expert ECG interpretation may be a dying art. Stewart Hart and Calvin Smith warned of the detail and precision that is required to analyze and interpret ECGs, and that this invaluable skill must be "practised regularly, systematically, and faithfully" [2].

Medical trainees are expected to learn a vast array of subjects and acquire countless clinical skills in the few short years that are dedicated to formal medical education. Only a fraction of this time is dedicated to formal ECG education and, unfortunately, opportunities for formal ECG training are lacking once these students enter the professional workforce [15]. In addition to the lack of formal ECG education available, there is an everlasting challenge for medical educators. Educators must develop a curriculum that is not only broad enough to capture the many complexities of ECG analysis, but also specific enough to provide learners with the details needed to be deemed competent in their evaluation [15]. A formal curriculum on ECG interpretation once trainees have entered the professional workforce also faces the constant challenge of accommodating the demanding resident schedule. 
These obstacles, in addition to countless others, have hindered formal ECG education and have led to an ever-growing population of healthcare professionals who do not feel confident or are not competent in their ECG analysis abilities. For these reasons, it does not come as a surprise that many have come to rely on the computerized ECG interpretation rather than their own skills for ECG analysis. The solution to reviving the lost art of ECG interpretation must begin at the most basic level of education in early pre-clinical years of medical school and gradually build on this foundation until competency is achieved.

Although the computerized ECG interpretation algorithms are regularly improved, their diagnostic accuracy is limited [14]. Computer ECG analysis has been criticized for many years. In 1991, a systemic assessment of computer-based ECG interpretation showed that the computer program's accuracy was $6.6 \%$ lower than the comparative cardiologists [14]. Additionally, the variability in diagnostic performance varied more greatly between computer models than between cardiologists [14].

Computerized ECG interpretation can contribute to diagnostic inaccuracies. The consequence of these inaccuracies is not limited only to simply a delay in diagnosis but may also have detrimental consequence from treatment delay. One of these detrimental delays can be seen in delay in diagnosis of STEMI, resulting in delaying the door to balloon time of coronary artery reperfusion. One study found a high rate of false negative results in computer diagnosis of STEMI advising against computerized ECG interpretation for such use [16] Given the detrimental consequence of false negative ECGs for STEMI, the need for clinician interpretation of final ECG diagnosis has become common practice in patients presenting with acute coronary syndrome symptom [17].

The necessity of clinician over-read of ECG in the diagnosis is essential and widely accepted among most healthcare institutions. One of the most relied upon features of the computerized ECG analysis is the measurement of intervals in the rhythm strip. The automated measurement of a QT interval is generally longer than the QT interval manually measured [14]. The over estimation of the QT interval may have significant implications on various cardiac and non-cardiac disease states and management of patients. Over estimation of the QT interval may lead to unnecessary dose adjustments or treatment changes due to the concern of QT interval prolongation. This can lead to suboptimal patient care and leave patients without the best treatment options due to the inaccurate interval estimation. Additionally, in families with long QT syndrome, an inaccurately recorded QT interval may lead to misdiagnosis.

The inaccuracies of ECG interpretation are not consistent across all disease states; the computer-based algorithm interpretation varies significantly in accuracy based on the underlying electrocardiographic rhythm. The positive predictive value of computer interpretation of sinus rhythm was $95 \%$, but only $53.5 \%$ in non-sinus rhythms and uninterpretable in $2 \%$ of ECG tracings in one study of 2112 randomly selected ECGs [14]. Furthermore, the computer algorithm misinterpreted $75 \%$ of pacemaker rhythms in older studies, which has led to improvement in pacemaker algorithms [14]. Despite the wellestablished inaccuracies of computer interpretation of ECGs, this analysis remains trusted by many clinicians and relied on for diagnostic accuracy.

\section{AI-ECG Interpretation}

Advances in machine learning have enabled a potential avenue to improve comprehensive ECG interpretation. Multiple studies have demonstrated the ability of AIECG algorithms to not only perform detection of various arrhythmias from single-lead ECGs [17-19] but also provide comprehensive, human-like 12-lead ECG interpretation [4]. This is exemplified in Figure 1, where the AI-ECG interpretation provides a more thorough ECG interpretation when compared to the traditional computer-generated interpretation. Moreover, the AI-ECG interpretation of the ECG in Figure 1 accurately identifies premature atrial complexes, while the traditional computer-generated interpretation labels the premature atrial complex as sinus arrhythmia and overestimates the QT. Figure 2 also demonstrates the AI-ECG model identifying first-degree AV block that was not labeled by 
the traditional computer ECG interpretation. When the performance of an AI-ECG algorithm was evaluated by cardiac electrophysiologists and compared to an existing standard automated computer program used in clinical practice, it was noted to outperform it and better approximate expert over-reads than an existing, widely used computerized ECG interpretation software [5].

The AI-ECG algorithm was applied to ECGs with 66 diagnoses codes by Kashou et al. [4]. In this study, the AI-ECG algorithm was able to generate diagnoses codes consistent with the interpretations of cardiologists. The model performed well for a wide range of ECG diagnoses codes including rhythm, conduction, ischemia, and waveform morphology [4]. Kashou et al. went on to emphasize that the abilities of the AI-ECG algorithm will continue to improve its interpretation skills as more high-quality raw data is incorporated into its algorithm [4].

In a similar study, the accuracy of AI-ECG was compared to traditional computer interpretation and final clinician interpretation of 500 ECGs [5]. Expert over-reading cardiologist in this study rated the interpretation of the traditional computer interpretation, AI-ECG interpretation, and clinician interpretation as unacceptable, acceptable, or ideal based on the accuracy of the interpretation. The results of this study showed that $202(13.5 \%)$ of the traditional computer interpreted, $123(8.2 \%)$ of the AI-ECG interpreted, and $90(6.0 \%)$ of the clinician interpreted ECGs were deemed as unacceptable and required edits [5]. Conversely, 958 (63.9\%) of the traditional computer interpreted, $1058(70.5 \%)$ of the AI-ECG interpreted, and $1118(74.5 \%)$ of the clinician interpreted ECGs were considered ideal and did not require edits [5]. Analysis on this data demonstrated that AI-ECG algorithms outperformed traditional computerized interpretation. In addition, AI-ECG algorithms were a better approximation of expert cardiologist over-read [5]. This further exemplifies the suggestion that AI-ECG interpretation may serve as an alternative, more accurate ECG interpretation compared to traditional computer algorithms.

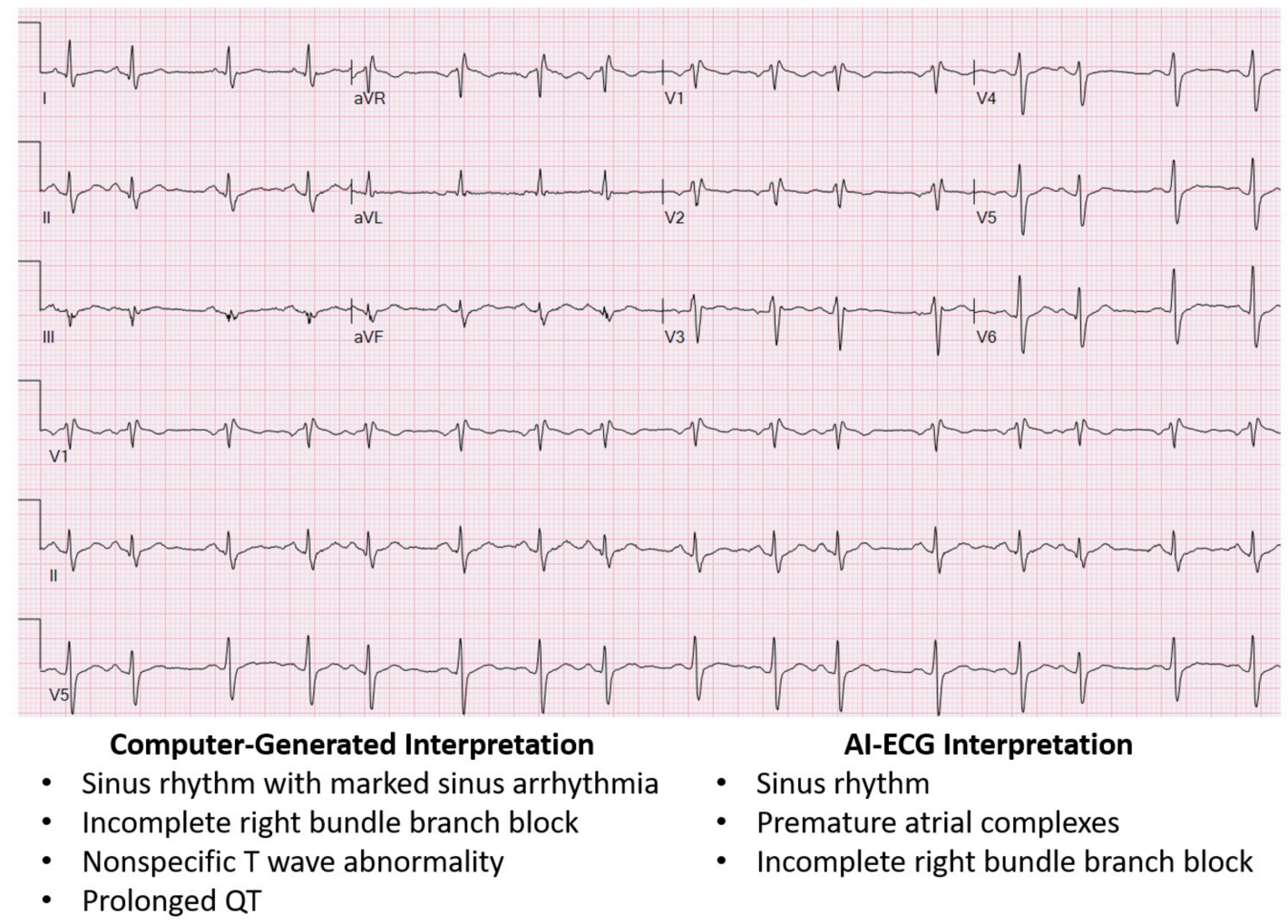

Figure 1. ECG showing traditional computer-generated interpretation versus AI-ECG interpretation. The AI-ECG interpretation provides a more accurate interpretation of the ECG, while the traditional computer-generated interpretation mislabels sinus arrhythmia for premature atrial complexes and overestimates the QT interval. 


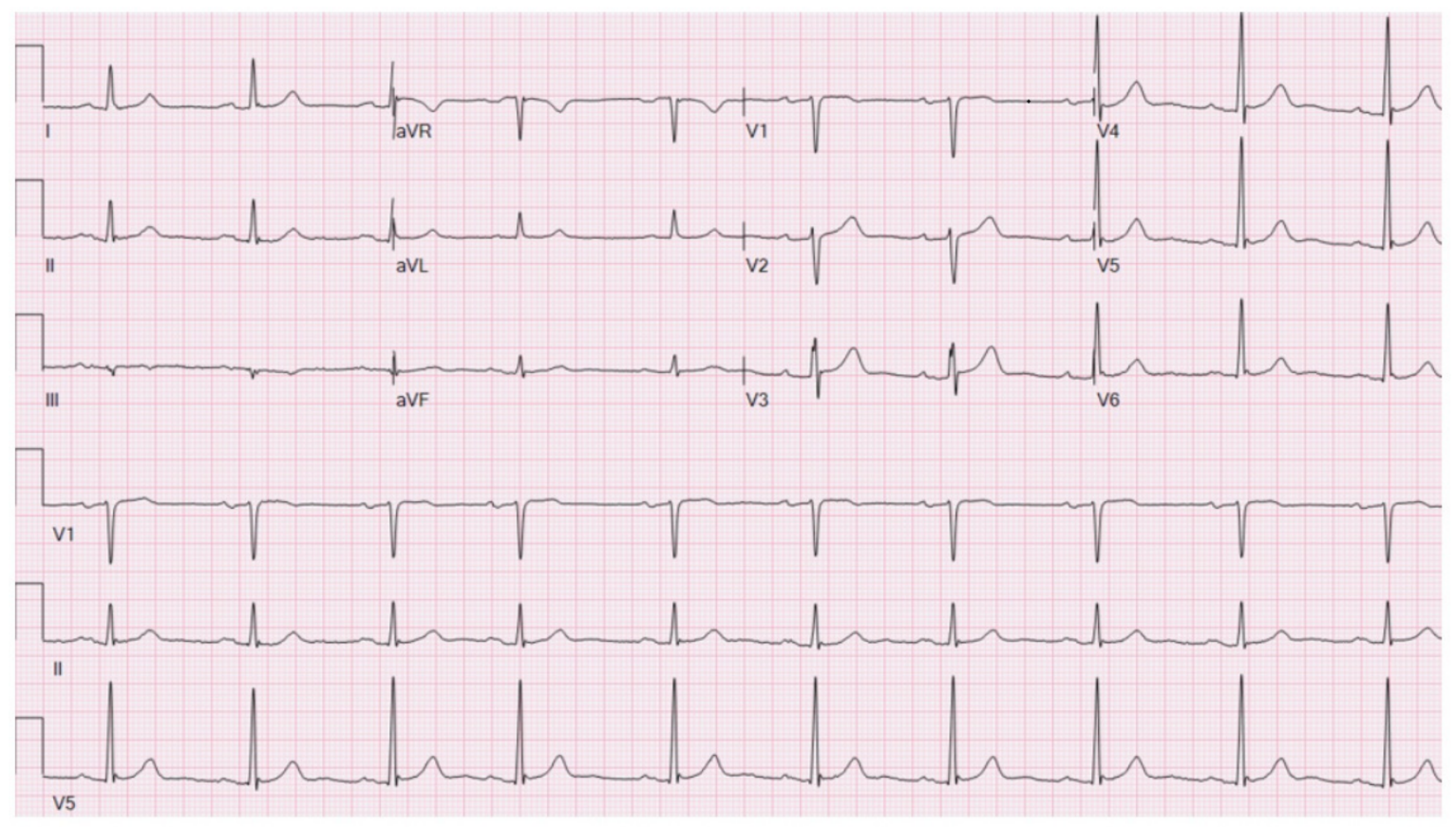
Computer-Generated Interpretation
- Sinus bradycardia
- Otherwise normal ECG

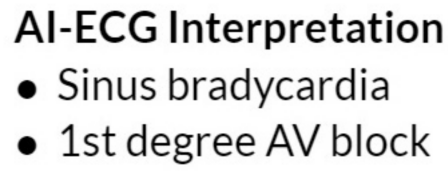

Figure 2. ECG showing traditional computer-generated interpretation versus AI-ECG interpretation. The AI-ECG interpretation provides a more specific and accurate interpretation of the ECG, while the computer-generated interpretation does not identify the first-degree AV block. While the AI-ECG algorithm is able to identify the AV conduction defect, it does not report a PR interval duration like traditional computer-generated algorithms.

It is still unclear how such an AI-ECG algorithm capable of comprehensive 12-lead ECG interpretation would perform against other conventional computer-devised models, if its performance would be preserved in various populations, and how it would be used in the clinical setting. Figure 3 illustrates a potential clinical workflow incorporation of AI-ECG into the clinical workflow including its ability to enhance AI-ECG interpretation as well as incorporate a novel AI-ECG prediction variable to improve the delivery of patient care. Additional research is required to improve AI-ECG model interpretation accuracy, better understand how to seamlessly incorporate it into clinical practice, and to expand access in resource-scarce regions.

The importance of advancing and improving AI-ECG algorithms can be seen and highlighted in institutions where there is a high volume of ECGs recorded daily. This is exemplified at our institution where over 100 trained ECG technologists are always present and reading ECGs with physician oversight. The vast amount of resources that such an ECG lab requires can be mitigated with the incorporation of advancing AI-ECG interpretations. AI-ECG interpretation will allow for the potential to increase overall accuracy and decrease the demand on ECG technologists and physicians. 


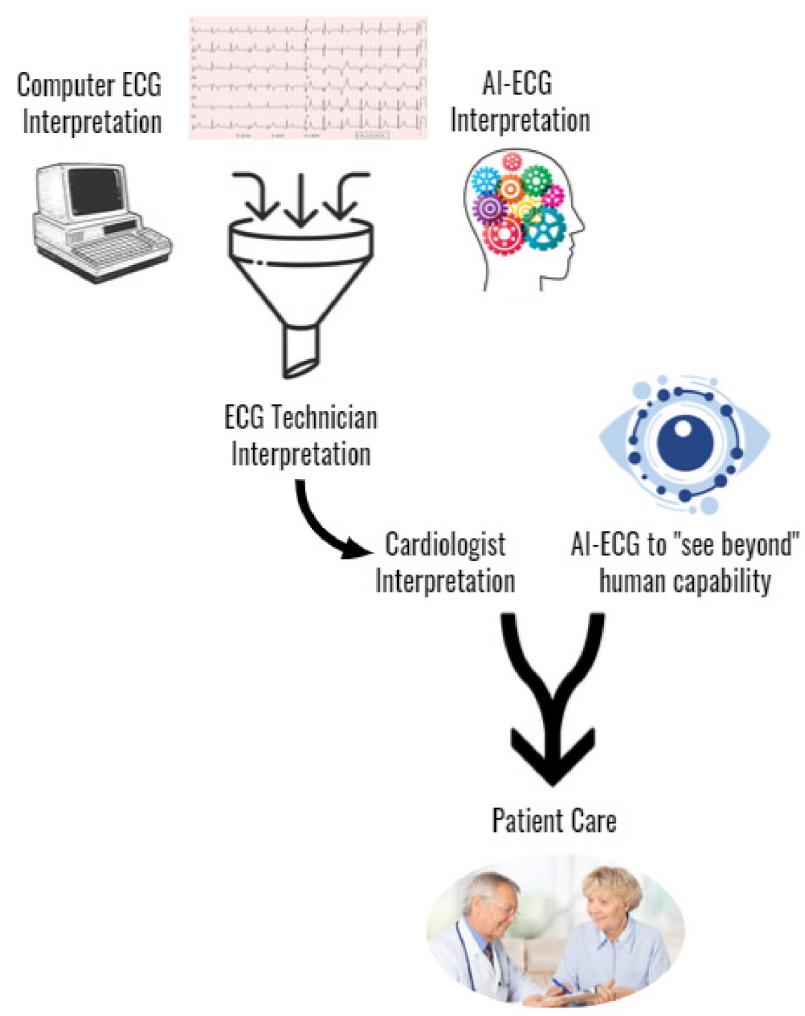

Figure 3. Proposed incorporation of AI-ECG into the clinical workflow.

\section{A Look Ahead}

With the emergence of mobile cardiac monitoring modalities into the marketplace, the ability to obtain electrocardiographic signals at any moment is now feasible. This instantaneous capture of high-quality cardiac signals can help personalize, expedite, and optimize patient care. Patients experiencing intermittent palpitations can record their heart's rhythm at the onset of symptoms on their own personal device without having to consult a medical provider to obtain a remote monitoring device. These signals could then be displayed at an in-person appointment or transmitted to a medical provider to help better understand if an underlying arrhythmia is contributing to the patient's symptoms and whether further investigation and/or a change in management is warranted. This will not only save the patient and healthcare institution time and finances but also be used to capture rare and intermittent symptoms that may not necessarily be captured over the standard 24 to $48 \mathrm{~h}$ of remote monitoring.

Despite these advances in data collection, there remain barriers as to how to ideally transmit, interpret, and use this ECG data clinically. With the ever-growing list of devices, a user-friendly means for patients to transmit the data to a provider is needed. Such a platform that ingests the ECG data must be able to accept various devices and be able to scale as new innovative technologies come to market. Additionally, quality controls must be in place to ensure the data are acceptable for clinical use. Lastly, and perhaps a future daunting some clinicians, is the foreseeable burden of data and increasing patient expectations. Actions must be in place to help alleviate the burden, compensate their work, and allow innovation to benefit all parties.

\section{Conclusions}

The performance and interpretation of the ECG is vital to the practice of medicine. In fact, this century-old diagnostic tool is experiencing a renaissance as novel technologies and potential clinical utility come to light. With the ever-growing utility of the various cardiac devices becoming available, it is more evident than ever that the ECG literacy is an essential skill. The commonly accepted conventional computerized ECG interpretation 
algorithms have many pitfalls and require physician oversight. As AI-ECG algorithms continue to improve their diagnostic accuracy, there is the possibility to improve ECG interpretation accuracy, improve clinical workflow, and better serve under-resourced areas. While advances and refinement in AI-ECG algorithms may help minimize ECG interpretation inaccuracies, ongoing efforts to improve ECG education for all medical providers will remain essential to provide high-quality patient care.

Author Contributions: Conceptualization, A.H.K. and P.A.N.; writing-original draft preparation, N.R. and A.H.K.; writing-review and editing, A.H.K. and P.A.N.; visualization, A.H.K.; supervision, A.H.K. and P.A.N. All authors have read and agreed to the published version of the manuscript.

Funding: This work was supported by the Department of Cardiovascular Medicine at Mayo Clinic in Rochester, MN. The authors also acknowledge support by NIH T32 HL007111.

Institutional Review Board Statement: Not applicable.

Informed Consent Statement: Not applicable.

Conflicts of Interest: A.H.K. and P.A.N. are potential beneficiaries of intellectual property discussed in this article. The remaining author declares no conflicts of interest.

\section{References}

1. Reichlin, T.; Abächerli, R.; Twerenbold, R.; Kühne, M.; Schaer, B.; Müller, C.; Sticherling, C.; Osswald, S. Advanced ECG in 2016: Is there more than just a tracing? Swiss. Med. Wkly. 2016, 146, w14303. [CrossRef] [PubMed]

2. Lüderitz, B.; de Luna, A.B. The history of electrocardiography. J. Electrocardiol. 2017, 50, 539. [CrossRef] [PubMed]

3. Fye, W.B. A history of the origin, evolution, and impact of electrocardiography. Am. J. Cardiol. 1994, 73, 937-949. [CrossRef]

4. Kashou, A.H.; Ko, W.-Y.; Attia, Z.I.; Cohen, M.S.; Friedman, P.A.; Noseworthy, P.A. A comprehensive artificial intelligence-enabled electrocardiogram interpretation program. Cardiovasc. Digit. Health J. 2020, 1, 62-70. [CrossRef]

5. Kashou, A.H.; Mulpuru, S.K.; Deshmukh, A.J.; Ko, W.-Y.; Attia, Z.I.; Carter, R.E.; Friedman, P.A.; Noseworthy, P.A. An artificial intelligence-enabled ECG algorithm for comprehensive ECG interpretation: Can it pass the 'Turing test'? Cardiovasc. Digit. Health J. 2021, 2, 164-170. [CrossRef]

6. Gosse, P.; Jan, E.; Coulon, P.; Cremer, A.; Papaioannou, G.; Yeim, S. ECG detection of left ventricular hypertrophy: The simpler, the better? J. Hypertens. 2012, 30, 990-996. [CrossRef] [PubMed]

7. Ang, K.P.; Nordin, R.B.; Lee, S.C.Y.; Lee, C.Y.; Lu, H.T. Diagnostic value of electrocardiogram in cardiac tamponade. Med. J. Malaysia 2019, 74, 51-56. [PubMed]

8. McNamara, N.; Ibrahim, A.; Satti, Z.; Ibrahim, M.; Kiernan, T.J. Acute pericarditis: A review of current diagnostic and management guidelines. Future Cardiol. 2019, 15, 119-126. [CrossRef] [PubMed]

9. Clementy, N.; Fourquet, A.; Andre, C.; Bisson, A.; Pierre, B.; Fauchier, L.; Babuty, D.; Angoulvant, D. Benefits of an early management of palpitations. Medicine 2018, 97, e11466. [CrossRef] [PubMed]

10. Martínez-Losas, P.; Higueras, J.; Gómez-Polo, J.C.; Brabyn, P.; Ferrer, J.M.; Cañadas, V.; Villacastín, J.P. The influence of computerized interpretation of an electrocardiogram reading. Am. J. Emerg. Med. 2016, 34, 2031-2032. [CrossRef] [PubMed]

11. Novotny, T.; Bond, R.; Andrsova, I.; Koc, L.; Sisakova, M.; Finlay, D.; Guldenring, D.; Spinar, J.; Malik, M. The role of computerized diagnostic proposals in the interpretation of the 12-lead electrocardiogram by cardiology and non-cardiology fellows. Int. J. Med. Inform. 2017, 101, 85-92. [CrossRef] [PubMed]

12. Bogun, F.; Anh, D.; Kalahasty, G.; Wissner, E.; Bou Serhal, C.; Bazzi, R.; Weaver, W.D.; Schuger, C. Misdiagnosis of atrial fibrillation and its clinical consequences. Am. J. Med. 2004, 117, 636-642. [CrossRef]

13. Anh, D.; Krishnan, S.; Bogun, F. Accuracy of electrocardiogram interpretation by cardiologists in the setting of incorrect computer analysis. J. Electrocardiol. 2006, 39, 343-345. [CrossRef] [PubMed]

14. Schläpfer, J.; Wellens, H.J. Computer-Interpreted Electrocardiograms: Benefits and Limitations. J. Am. Coll. Cardiol. 2017, 70, 1183-1192. [CrossRef]

15. Kashou, A.; May, A.; DeSimone, C.; Noseworthy, P. The essential skill of ECG interpretation: How do we define and improve competency? Postgrad. Med. J. 2020, 96, 125-127. [CrossRef] [PubMed]

16. Garvey, J.L.; Zegre-Hemsey, J.; Gregg, R.; Studnek, J.R. Electrocardiographic diagnosis of ST segment elevation myocardial infarction: An evaluation of three automated interpretation algorithms. J. Electrocardiol. 2016, 49, 728-732. [CrossRef] [PubMed]

17. Acharya, U.R.; Fujita, H.; Lih, O.S.; Hagiwara, Y.; Tan, J.H.; Adam, M. Automated detection of arrhythmias using different intervals of tachycardia ECG segments with convolutional neural network. Inf. Sci. 2017, 405, 81-90. [CrossRef] 
18. Xiong, Z.; Stiles, M.K.; Zhao, J. Robust ECG signal classification for detection of atrial fibrillation using a novel neural network. In Proceedings of the 2017 Computing in Cardiology (CinC), Rennes, France, 24-27 September 2017; pp. 1-4.

19. Hannun, A.Y.; Rajpurkar, P.; Haghpanahi, M.; Tison, G.H.; Bourn, C.; Turakhia, M.P.; Ng, A.Y. Cardiologist-level arrhythmia detection and classification in ambulatory electrocardiograms using a deep neural network. Nat. Med. 2019, 25, 65-69. [CrossRef] [PubMed] 